\title{
Chemical Transformations of Eremanthine. Synthesis of Micheliolide and 1(R),10(R)-Dihydromicheliolide
}

\author{
José C. F. Alves* and Edna C. Fantini ${ }^{\dagger}$ \\ Departamento de Química, Instituto de Ciências Exatas, \\ Universidade Federal Rural do Rio de Janeiro, \\ 23890-970 Seropédica-RJ, Brazil
}

2005, vol. 16, No 4, 749-755.

Page 749, abstract in Portuguese, line 4, the correct proportion is: $(8: 1)$.

Page 749, abstract in English, line 3, the correct proportion is: $(8: 1)$.

Page 751, column 2, line 20, the correct proportion is: 8:1.

Page 751, scheme 2, the correct proportion between the compounds 7 and 6 is: (1:8).
Page 752, column 1, line 4, the correct overall yield of micheliolide (9) is: $36 \%$.

Page 754, column 1, first paragraph, line 17, the correct coupling constant at $\delta 5.48$ is: $J 3.0 \mathrm{~Hz}$.

Page 754, column 1, second paragraph, line 17, the correct coupling constant at $\delta 5.41$ is: $J 3.2 \mathrm{~Hz}$.

Page 754, column 2, reference 10, the following reference should be added: Lima, P. D. D. B.; Garcia, M.; Rabi, J. A.; J. Nat. Prod. 1985, 48, 986. 